\title{
Estimation of the friction velocity in stably stratified boundary-layer flows over hills
}

Article

Accepted Version

Argaín, J. L., Miranda, P. M. A. and Teixeira, M. A. C. (2009) Estimation of the friction velocity in stably stratified boundarylayer flows over hills. Boundary-Layer Meteorology, 130 (1). pp. 15-28. ISSN 1573-1472 doi:

https://doi.org/10.1007/s10546-008-9329-3 Available at https://centaur.reading.ac.uk/29244/

It is advisable to refer to the publisher's version if you intend to cite from the work. See Guidance on citing.

Published version at: http://dx.doi.org/10.1007/s10546-008-9329-3

To link to this article DOI: http://dx.doi.org/10.1007/s10546-008-9329-3

Publisher: Springer

All outputs in CentAUR are protected by Intellectual Property Rights law, including copyright law. Copyright and IPR is retained by the creators or other copyright holders. Terms and conditions for use of this material are defined in the End User Agreement.

\section{www.reading.ac.uk/centaur}

\section{CentAUR}

Central Archive at the University of Reading 
Reading's research outputs online 


\title{
Estimation of the friction velocity in stably stratified boundary-layer flows over hills
}

\author{
José Luis Argaín ${ }^{1}$ \\ University of Algarve, Department of Physics, Faro, Portugal
}

Pedro M. A. Miranda, Miguel A. C. Teixeira

University of Lisbon, CGUL, IDL, Lisbon, Portugal

\begin{abstract}
In this paper a method is suggested for the calculation of the friction velocity for stable turbulent boundary-layer flow over hills. The method is tested using a continuous upstream mean velocity profile compatible with the propagation of gravity waves, and is incorporated into the linear model of Hunt, Leibovich and Richards with the modification proposed by Hunt, Richards and Brighton to include the effects of stability, and the reformulated solution of Weng for the nearsurface region. Those theoretical results are compared with results from simulations using a nonhydrostatic micro-mesoscale two-dimensional numerical model, and with field observations for different values of stability. These comparisons show a considerable improvement in the behaviour of the theoretical model when the friction velocity is calculated using the method proposed here, leading to a consistent variation of the boundary layer structure with stability, and better agreement with observational and numerical data.
\end{abstract}

Keywords: friction velocity, stable stratification, flow speed-up, linear theory, middle-layer height

\footnotetext{
${ }^{1}$ Corresponding author: José Luís Argain, Departamento de Física, Faculdade de Ciências e Tecnologia, Universidade do Algarve, Campus de Gambelas, 8005-139 Faro, Portugal, Phone:00351289800900, Fax: 003512898000066, Email: jargain@ualg.pt
} 


\section{Introduction}

Most studies of turbulent boundary layer flow over complex terrain consider nearly unstratified conditions. Indeed, the description of the turbulent boundary layer (BL), using either numerical or theoretical models, becomes more difficult with increasing stability, not only because of the growing importance of nonlinear effects, but also because of the changes in the boundary layer structure and its interaction with the terrain. Such changes must be carefully taken into account in the calculation of the parameters that characterize the turbulent BL.

One of the first theoretical BL models of flow over hills was developed by Jackson and Hunt (1975) and subsequently improved by Hunt et al. (1988a) (HLR). Hunt et al. (1988b) (HRB) incorporated a modification in the HLR model to take into account the effects of stratification. Belcher et al. (1993), using an approach similar to that of HLR, determined the form drag exerted on hills, and Belcher and Wood (1996) included the effect of stratification in the calculation of that quantity. Weng (1989), after performing a comparison with observations, found that the HLR solution overpredicts the velocity perturbation, especially near the surface, and suggested a modification that corrected this problem. On the other hand, to allow for the propagation of gravity waves outside the BL, Weng et al. (1997) proposed a new continuous wind profile as an alternative to the standard log-linear profile based on Monin-Obukhov (M-O) similarity theory.

Many studies have been dedicated to the determination of the vertical fluxprofile relationships in the BL, e.g., Businger et al. (1971), Dyer (1974), Högström (1996) and Yagüe et al. (2006). Barker and Baxter (1975) derived formulas for the surface layer fluxes that can be applied as lower boundary conditions in BL models and Louis (1979) developed a parametric model for the representation of the vertical eddy fluxes in atmospheric models. Klug (1967) used the integrated form of the KEYPS profiles (Paulson 1970) to derive expressions for the friction velocity and the vertical heat flux, and Ting and Hay (1975), based on the free convection wind and temperature profiles, improved Klug's prediction. Weber (1999), by means of wind measurements, compared the friction velocities resulting from different definitions, and Akylas et al. (2003) studied the concept of a minimum friction velocity.

In his theoretical calculations, Weng (1997) employed a friction velocity independent of the atmospheric stability. On the other hand, his plots of the flow speed-up as a function of the Monin-Obukhov length using his continuous velocity profile display a jump at low stability values that is not conveniently explained. In the present study, these seemingly unconnected aspects are related, and a simple method is suggested for estimating the friction velocity, which corrects this problem. Theoretical predictions are produced using linear theory, i.e. the HLR model, combined with the modifications of HRB, Weng (1989) and Weng et al. (1997). Numerical simulations are carried out using the nonhydrostatic, two-dimensional model FLEX, developed by Argain (2003). The theoretical model is applied in two cases, where: a) the friction velocity is kept constant, and b) the friction velocity is calculated for each stability regime, employing the method proposed here. The performance of the theoretical model is evaluated by comparison with the numerical model, and with field data reported by Coppin et al. (1994).

This paper is organized as follows: in section 2 the theoretical model of flow over hills is briefly reviewed, and in section 3 the method proposed for estimating 
the friction velocity is described. In sections 4 and 5 we give a brief description of the numerical model and of the experimental data. Section 6 contains a presentation and discussion of the results and in section 7 the main conclusions are summarized.

\section{Theoretical model}

The M-O similarity theory predicts that the non-dimensional vertical gradients of mean velocity $U(z)$, and mean potential temperature $\theta(z)$ are universal functions of height normalized by a length scale $L_{m o}$, in the form

$$
\begin{aligned}
& \Phi_{m}\left(\frac{z}{L_{m o}}\right)=\frac{\kappa z}{u_{*}} \frac{\partial U}{\partial z}, \\
& \Phi_{h}\left(\frac{z}{L_{m o}}\right)=\frac{\kappa z}{\theta_{*}} \frac{\partial \theta}{\partial z} .
\end{aligned}
$$

Using the formula proposed by Dyer (1974) for stable conditions $\left(z / L_{m o}>0\right)$

$$
\Phi_{h}=\Phi_{m}=1+\beta \frac{z}{L_{m o}} .
$$

Equations 1 and 2 can be integrated from the roughness height $z_{0}$ to the height $z+z_{0}$, to obtain

$$
\begin{gathered}
\theta(z)=\theta_{0}+\frac{\theta_{*}}{\kappa}\left[\ln \left(\frac{z}{z_{0}}+1\right)+\beta \frac{z}{L_{m o}}\right], \\
U(z)=\frac{u_{*}}{\kappa}\left[\ln \left(\frac{z}{z_{0}}+1\right)+\beta \frac{z}{L_{m o}}\right],
\end{gathered}
$$

where $\kappa$ is the von Kármán constant $(\kappa=0.41), \beta$ is a constant ( $\beta=5$ in Dyer 1974) and $\theta_{0}$ is the potential temperature at the roughness height $z_{0}$. Note that Eq. 3 should not be used for $z / L_{m o}>1$ (Yagüe et al. 2006). The scaling velocity $u_{*}$ (the friction velocity) and temperature $\theta *$ are defined from the vertical eddy fluxes of momentum and sensible heat, as $u_{*}=\sqrt{\left|\overline{w^{\prime} u^{\prime}}\right|_{0}}$ and $\theta_{*}=-\left(\overline{\theta^{\prime} w^{\prime}}\right)_{0} / u_{*}\left(u_{*}\right.$ and $\theta_{*}$ are fundamental scaling parameters of this similarity theory). The quantity $L_{m o}$ represents the $\mathrm{M}-\mathrm{O}$ length scale, which is defined as

$$
L_{m o}=-\frac{\theta_{0} u_{*}^{3} / \kappa}{g\left(\overline{\theta^{\prime} w^{\prime}}\right)_{0}}=\frac{\theta_{0} u_{*}^{2}}{g \theta_{*} \kappa} .
$$

In the HLR model, the flow field is divided into different regions according to different flow dynamics: in an inner region, of depth $h_{i}$, the acceleration, pressure gradient force and divergence of the turbulent momentum flux make comparable contributions to the streamwise momentum balance. In a middle region, of depth $h_{m}$, defined by $\left(h_{i}<z<h_{m}\right)$, it is assumed that the flow is inviscid but rotational, and in an outer region $\left(z>h_{m}\right)$ the flow is considered both inviscid and irrotational. The linearized HLR model connects the variables of each region using the method of matched asymptotic expansions, providing a complete description of the flow field. 
According to HLR and Hunt and Richards (1984), the implicit equation for estimating $h_{i}$ in the stability-modified inner region is

$$
h_{i} U\left(h_{i}\right) / u_{*}=2 \kappa^{2} L,
$$

where $U\left(h_{i}\right)$ is the upstream velocity evaluated in $z=h_{i}$ and $L$ is the characteristic horizontal length scale (defined as the distance from the crest of the hill to the upstream point where the elevation is half of the maximum height, $h_{0}$ ). In the inviscid regions, the flow behaviour is described by Scorer's equation which, for two-dimensional flow, has the following form

$$
\left\{\frac{\partial^{2}}{\partial x^{2}}+\frac{\partial^{2}}{\partial z^{2}}+\frac{N^{2}}{U^{2}}-\frac{1}{U} \frac{d^{2} U}{d z^{2}}\right\} W^{\prime}=0 .
$$

In this equation, $W^{\prime}$ represents the vertical velocity perturbation and $N$ is the Brunt-Väisälä frequency, defined as

$$
N^{2}=\frac{g}{\theta} \frac{\partial \theta}{\partial z}
$$

According to HLR, the middle layer is defined as the lower part of the outer region where the term involving the curvature of the velocity profile, $U^{-}$ ${ }^{1}\left(d^{2} U / d z^{2}\right) W^{\prime}$, balances the second derivative term $d^{2} W^{\prime} / d z^{2}$. However, Weng (1997) showed that, as the flow becomes more stable, the buoyancy term, $\left(N^{2} / U^{2}\right) W^{\prime}$, should also be taken into account in the determination of the middle layer height, $h_{m}$. Thus, this author proposed a more general expression for the estimation of $h_{m}$, namely:

$$
\left|U^{-1}\left(h_{m}\right) d^{2} U\left(h_{m}\right) / d z^{2}\right|=L^{-2}+\left(N\left(h_{m}\right) / U\left(h_{m}\right)\right)^{2} .
$$

In their paper about stable stratification effects on form and wave drag, Belcher and Wood (1996) showed that the height where buoyancy destruction and shear production of turbulent kinetic energy $(E)$ have comparable magnitude is much higher than the inner layer depth (when $h_{i} / L_{m o}<<1$ ), so the flow perturbations in the inner region are formally the same as for the equations that govern neutral flow. So, even though the length scales and velocity profiles are different in the inner region, the HLR solution in stable and neutral flow has the same form. The solutions for the various layers are obtained and asymptotically matched in Fourier space. According to HLR, the streamwise velocity perturbation in wave number domain is given by

$$
\widetilde{u}^{\prime}(k, z)=-\frac{U^{2}\left(h_{m}\right)}{U\left(h_{i}\right)} \widetilde{p}_{s}^{\prime} \varphi\left(k, z, z_{0}, h_{i}, L\right),
$$

where $k$ is the horizontal wave number and $\tilde{p}_{s}{ }_{s}$ represents the Fourier transform of the pressure perturbation at the surface. In the HLR model the perturbation pressure field, $p_{s}^{\prime}$, is determined by the solution in the outer layer and is normalized by $\rho U^{2}\left(h_{m}\right)$. The HLR model is valid for orographies whose ratio $h_{0} / L$ is approximately less than 0.3 . In order to take the effects of the stratification into account, the pressure $\widetilde{p}_{s}^{\prime}$ is calculated here according to the modifications proposed by HRB. 


\subsection{Flow speed-up}

The ratio of the wind perturbation $\left(u^{\prime}(x, z)=u(x, z)-U(z)\right)$ to the mean wind at a point far upstream, at a fixed height $z$, is called the fractional speed-up ratio $(\Delta S)$ and can be written as

$$
\Delta S(x, z)=\frac{u^{\prime}(x, z)}{U(z)} .
$$

Calculating the inverse Fourier transform of Eq. 11, $\Delta S$ is obtained for the inner region as

$$
\Delta S(x, z)=\frac{U^{2}\left(h_{m}\right)}{U\left(h_{i}\right) U(z)} \zeta\left(x, z_{0}\right),
$$

where $\zeta\left(x, z_{0}\right)$ is a function of order one that depends on the shape of the hill, surface roughness and stratification. In an analogous way, in the middle layer $\Delta S$ can be expressed as (Coppin et al. 1994)

$$
\Delta S(x, z)=\frac{U^{2}\left(h_{m}\right)}{U^{2}(z)} \xi(x),
$$

where, again, the function $\xi(x)$ is of order one and takes into account the shape of the hill. Since in the middle layer $z>h_{i}$ and $U(z)>U\left(h_{i}\right)$ for the M-O velocity profile of Eq. 5, comparison of Eqs. 13 and 14 reveals that $\Delta S$ in the inner layer is larger than in the middle layer.

Weng (1989) found that the HLR solution, given by Eq. 11, overpredicts field observations, particularly near the surface, and proposed to replace the complex function $\varphi\left(k, z, z_{0}, h_{i}, L\right)$ in Eq. 11 by a new function $\psi\left(k, z, z_{0}, h_{i}, L\right)$. This new function ensures that $u^{\prime}(x, z) \rightarrow 0$ as $z \rightarrow 0$ and that, as in the HLR solution, $u^{\prime}(x, z)$ matches with the middle layer solution as $z$ increases. Weng (1989) validated this modified solution against observations from the Askervein Hill Project reported by Taylor and Teunissen (1985) (which has been used as a test case for numerous authors - see, for example, Lopes et al. 2007) and obtained significant improvement by comparison with the original HLR model.

\section{Estimation of the friction velocity}

The wind profile given by Eq. 5 should not be used in simulations of the propagation of internal gravity waves because $U(z)$ grows indefinitely as $z \rightarrow \infty$. If $F r_{h}$, the horizontal Froude number, is defined as $F r_{h}=U_{\infty} k / N_{\infty}$, where $k$ represents the horizontal wave number of the perturbation being considered and $U_{\infty}=U(\infty)$, this wind profile would always lead to $F r_{h}>1$. In this case, gravity wave perturbations cannot propagate to the outer region.

To circumvent this problem, Weng et al. (1997) proposed a continuous mean velocity profile that matches a constant free stream velocity $U_{\infty}$ for large $z$ but still tends to the log-linear form close to the surface, as the standard M-O profile:

$$
U(z)=\frac{1}{2}\left[g_{1}(z)+g_{2}(z)+U_{\infty}+\sqrt{\left(g_{1}(z)+g_{2}(z)-U_{\infty}\right)^{2}+c_{0}^{2}}\right] .
$$

Here $g_{1}(z)$ is equal to $U(z)$ in Eq. 5 and $g_{2}(z)$ has the following form 


$$
g_{2}(z)=u_{*}\left[c_{1}+c_{2} \ln \left(\frac{z}{z_{0}}+1\right)+c_{3}\left(\frac{z}{z_{0}}+1\right)^{\sigma}\right] .
$$

The constants $c_{1}, c_{2}$ e $c_{3}$ depend on the values of $\beta, L_{m o}, z_{0}, U_{\infty}, u_{*}$ and on a constant $\mathrm{c}_{0}$ (see Weng et al. 1997). The velocity profile of Eq. 15 can be varied by adjusting the constants $c_{0}$ and $\sigma$. Being continuous, this profile can be used in theoretical models where it is necessary to calculate derivatives of the wind velocity, and prevents reflections at shear discontinuities in numerical simulations. When $U(z)$ is calculated using Eq. 15, if $z \rightarrow \infty$ then $U(z) \rightarrow U_{\infty}=$ const.

Weng (1997) obtained considerable improvements in the performance of the HLR model by using the HRB and Weng (1989) modifications and by calculating $h_{m}$ through Eq. 10, rather than using the expression suggested by HRL or HRB. These improvements were accomplished using the log-linear velocity profile, Eq. 5. However, when Weng (1997) replaced the log-linear profile by Eq. 15 in the HLR model including the other modifications mentioned above, he found that the results diverge significantly from the observations, particularly in the weak to moderate stability range. The reason for this discrepancy is that the graphs of $\Delta S$ as a function of $1 / L_{m o}$ display a spurious peak after an apparent discontinuity. This aspect, which was only mentioned briefly in Weng (1997), has not been conveniently explained.

We suggest here that this disagreement is due to the fact that in Weng (1997) the calculations are carried out assuming $u_{*}$ to be a constant, regardless of the different stability regimes considered. In fact, the BL structure, and in particular the value of $u_{*}$, strongly depend on the stability regime. According to Eqs. 7 and 10 , the velocity profile $U(z)$ has a decisive role in the calcultion of both $h_{i}$ and $h_{m}$, and hence in the determination of the BL depth, $h$.

Often, in analytical and numerical models used for studying the propagation of internal gravity waves, the potential temperature profile in the outer region is imposed by means of $N_{\infty}=$ const. and the velocity is set to a constant $U_{\infty}$. In order to have a wind profile that correctly describes the BL structure under these conditions, the value of $u_{*}$ must be such that, for a given value of $N(h)=N_{\infty}$, the approximate condition $U(h)=U_{\infty}$ is satisfied.

Next, we will describe a simple method to estimate $u_{*}$. The velocity profile $U(z)$ assumed in the derivation of this method is the well-known M-O log-linear profile of Eq. 5. The key assumption for relating the quantities $u_{*}$ and $L_{m o}$ is the following expression, suggested by Zilitinkevich (1972) for estimating the BL depth,

$$
h=c_{z s} \sqrt{u_{*} L_{m o} /|f|},
$$

where $f$ is the Coriolis frequency and $c_{z s}=0.4$.

The potential temperature profile, Eq. 4, is often observed to be a good approximation far above its theoretical limit $\left(\mathrm{z} \sim L_{m o}\right)$. Calculating $d \theta / d z(z \rightarrow \infty)$ from Eq. 4, and combining Eqs. 6 and 9, the following relation can be obtained

$$
N_{\infty}=u_{*} \beta^{1 / 2}\left(K L_{m o}\right)^{-1} .
$$

This provides a link between $L_{m o}$ and the Brunt-Väisälä frequency in the outer region $\left(N(h)=N_{\infty}\right)$. Using the preceding relation, Eq. 17 can be written as 


$$
h=c_{z s} u_{*} \beta^{1 / 4}\left(|f| \kappa N_{\infty}\right)^{-1 / 2} .
$$

The condition $U(h)=U_{\infty}$, combined with Eqs. 19 and 6 for $h$ and $L_{m o}$, respectively, yields

$$
u_{*}=\kappa U_{\infty}\left[\ln \left(\frac{c_{z s} u_{*}}{z_{0}} \sqrt{\frac{\beta^{1 / 2}}{|f| \kappa N_{\infty}}}+1\right)+\beta c_{z s} \sqrt{\frac{\kappa N_{\infty}}{|f| \beta^{-1 / 2}}}\right]^{-1} .
$$

This is an implicit equation for $u_{*}$. Thus, from known values of $U_{\infty}, z_{0}$ and $N_{\infty}$, the velocity $u_{*}$ can be estimated by calculating the solution of Eq. 20 . If the stability regime is known through the length scale $L_{m o}, u_{*}$ may alternatively be calculated from the expression

$$
u_{*}=\kappa U_{\infty}\left[\ln \left(\frac{c_{z s}}{z_{0}} \sqrt{\frac{u_{*}^{2}}{|f| L_{m o}}}+1\right)+c_{z s} \beta \sqrt{\frac{u_{*} L_{m o}}{|f|}}\right]^{-1} .
$$

On the other hand, in the neutrally-stratified case $h$ may be calculated using the expression of Rossby and Montgomery (1935)

$$
h=c_{z n} u_{*} /|f|,
$$

with $c_{z n}=0.3$ (Tjernstrüm and Smedman 1993). In this case, $u_{*}$ is estimated by solving the simpler equation

$$
u_{*}=\kappa U_{\infty}\left[\ln \left(\frac{c_{z n} u_{*}}{z_{0}|f|}+1\right)\right]^{-1},
$$

which produces satisfactory results in neutral conditions (not shown).

In the stably-stratified case, it will be considered that Eq. 20 is applicable if the $h$ calculated from Eq. 17 is smaller or equal to the $h$ given by Eq. 22. When these depths are equal, the value of $u_{*}$ estimated from Eq. 21 is used to obtain the corresponding upper limit of $L_{m o}$ for which this last equation may be applied. This value corresponds approximately to $L_{m o}=600 \mathrm{~m}$.

The method proposed here can be extended for use with an arbitrary stability function $\Phi_{h}$ by determining the following expression for $N_{\infty}^{2}$, analogous to Eq. 18, from Eqs. 2, 6 and 9:

$$
N_{\infty}^{2}=\left(\frac{u_{*}}{\kappa}\right)^{2} \frac{1}{h L_{m o}} \Phi_{h}\left(\frac{h}{L_{m o}}\right) .
$$

Using this relation and following the procedure explained above, $u_{*}$ can be determined in principle for any stability conditions. However, testing this method for non-stable stratifications is beyond the scope of the present study.

It is important to note that the present method does not require that $N(z)=$ const. and $U(z)=$ const. in the whole outer region but just that the values of these quantities are well defined at the height $z=h$, in order to obtain a realistic $u_{*}$. So, more complex wind profiles may be devised to replace Eq. 15, that allow, e.g., vertical wind shear outside the BL.

We recall that the theoretical model used in this study, and where the method described above is applied, is a combination of the HLR model with the HRB, Weng (1989) and Weng et al. (1997) modifications. 


\section{The numerical model (FLEX)}

The FLEX model is a nonhydrostatic micro- to mesoscale model developed and validated against experimental data by Argain (2003). This model has been used previously by Teixeira et al. $(2005,2008)$ to assess the behaviour of analytical mountain wave drag predictions by comparison with numerical results. The FLEX model includes a set of physical and numerical features that make it able to simulate mesoscale flows over arbitrary orography for any value of the static stability. The model uses a generalized orthogonal coordinate system and a local grid refinement technique that concentrates the computational effort where it is most necessary. Subgrid scale effects are modelled using a modified version of the standard $E-\varepsilon$ model, for atmospheric stably-stratified BL flows. Stability effects near the surface were included by means of a combination of wall functions and M-O similarity theory expressions. The solution technique is based on the SIMPLE algorithm of Patancar (1980).

\section{Experimental data}

In the spring of 1984 and summer of 1985, Coppin et al. (1994) carried out a field study of different types of flow over a somewhat isolated north-south oriented, quasi-two-dimensional ridge of uniform low surface roughness - the Cooper ridge, located north-west of Goulburn in New South Wales, Australia. The orography is located along a valley that forces the winds to blow on the hill predominantly from the west side. The windward face of the ridge (west side) can be well fitted using a simple bell-shaped profile $h(x)=h_{0} /\left(1+(x / L)^{2}\right)$ (where $h_{0}=115$ $\mathrm{m}$ and $L=400 \mathrm{~m}$ ). The lee side of the ridge falls away to about $0.5 h_{0}$ before rising to another broader ridge.

\section{Results and discussion}

As in Coppin et al. (1994) and Weng (1997), the effect of the lee side feature on the predicted $\Delta S$ is neglected in the theoretical model and all the calculations are carried out here using the $2 \mathrm{D}$ bell-shaped hill described above. The wind velocity in the outer layer is taken as $U_{\infty}=6 \mathrm{~m} / \mathrm{s}$, we assume that $u_{*}=0.2 \mathrm{~m} / \mathrm{s}$ when this quantity is prescribed (Weng 1997), and also that $z_{0}=0.05 \mathrm{~m}$ (Coppin et al. 1994). In the computations, the length $L_{m o}$ is varied from $20 \mathrm{~m}$ to $600 \mathrm{~m}$ which, by Eq. 18 (if $u_{*}$ is estimated from Eq. 20), corresponds to the following interval of BruntVäisälä frequencies: $N_{\infty} \in[0.0018,0.030] \mathrm{s}^{-1}$. Hence, the outer layer dimensionless height $\left(\hat{h}=N_{\infty} h_{0} / U_{\infty}\right)$ and the dimensionless length of the ridge $\left(\hat{L}=N_{\infty} L / U_{\infty}\right)$ are in the intervals $\hat{h} \in[0.035,0.6]$ and $\hat{L} \in[0.12,2]$, respectively. As the upper limit of the $\hat{L}$ interval is much less than 10 , all studied flows are clearly non-hydrostatic. Note that the flow with $\hat{h}=0.6$ is also quite non-linear, but remains yet within the limit of validity of the HRB analysis (approximately $\hat{h}<1$ ).

Figure 1 shows $u_{*}$ as a function of stability $\left(1 / L_{m o}\right)$, determined by the method proposed in the present study. Clearly, $u_{*}$ decreases significantly with increasing stability. In the less stable limit (largest $L_{m o}$ ) $u_{*}$ is approximately twice the value obtained in the more stable limit. This behaviour is to a certain extent intuitive, because stability inhibits turbulence. 


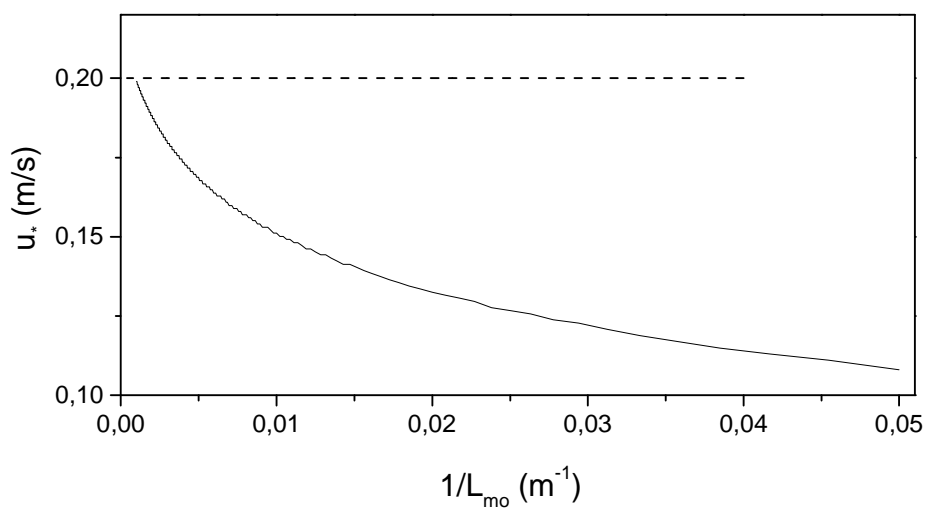

Fig. 1 Friction velocity $\left(u_{*}\right)$ as a function of $1 / L_{m o}$. Solid line: computed $u_{*}$; dashed line: prescribed $u_{*}\left(u_{*}=0.2 \mathrm{~m} / \mathrm{s}\right)$

The velocity profile given by Eq. 15, introduced by Weng (1997), contains a free parameter $c_{0}$. In the present work, the constant $c_{0}$ takes the value $c_{0}=0.025 U_{\infty}$, instead of the value $c_{0}=0.1 U_{\infty}$ employed by Weng (1997). Figure 2 shows a comparison between a discontinuous profile, in which $U(z)$ is calculated using the M-O profile, Eq. 5, for $z \leq 0.99 h$ and $U(z)=U_{\infty}$ for $z>0.99 h$, and the velocity profiles from Eq. 15 obtained with $c_{0}=0.1 U_{\infty}$ and $c_{0}=0.025 U_{\infty}$. The two stability values selected, $L_{m o}=20 \mathrm{~m}$ and $L_{m o}=600 \mathrm{~m}$, are extremes of the $L_{m o}$ interval considered. Both profiles were obtained using the value of $u_{*}$ computed by the proposed method (yielding $u_{*}=0.111 \mathrm{~m} / \mathrm{s}$ for $L_{m o}=20 \mathrm{~m}$ and $u_{*}=0.191 \mathrm{~m} / \mathrm{s}$ for $L_{m o}=600 \mathrm{~m}$ ). In order to better visualize the differences between the profiles, these were plotted only for $z / h>0.5$. For $z / h<0.5$ the mean velocity is practically coincident for the three profiles, because near the surface all of them follow M-O similarity. On the other hand, the profiles revealed a low sensitivity to the constant $\sigma$. In all the calculations performed, $\sigma=0.1$ is used, as suggested by Weng (1997). 


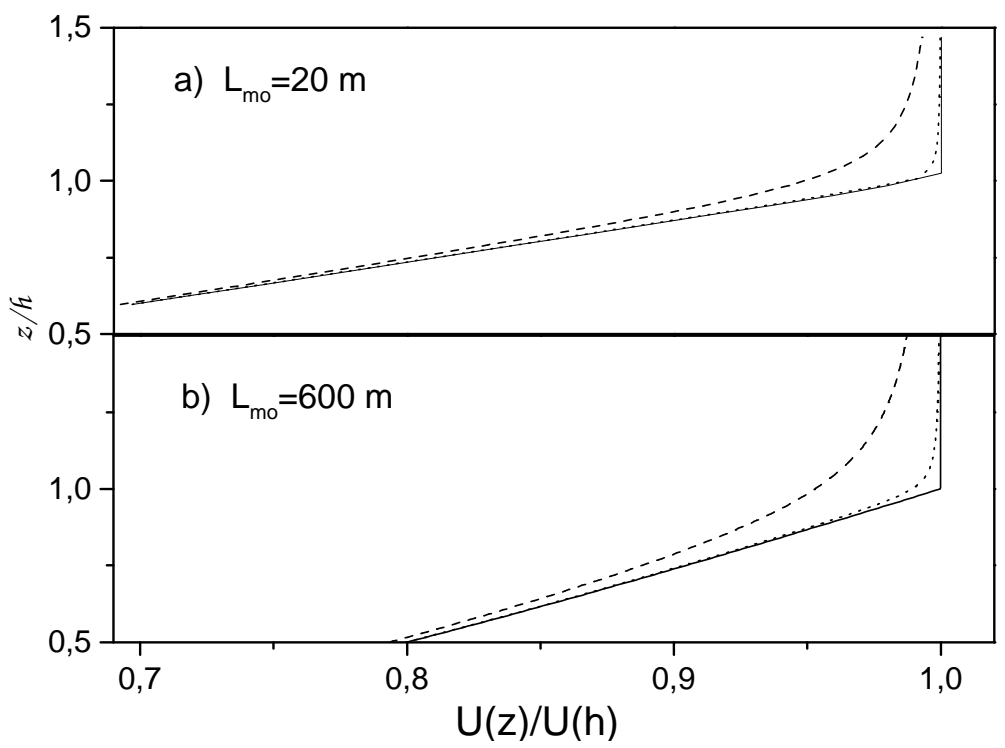

Fig. 2 Upstream velocity profiles, normalized by $U_{\infty}=U(h)$, for (a) $L_{m o}=20 \mathrm{~m}$, (b) $L_{m o}=600 \mathrm{~m}$. The height is normalized by $h$, estimated from Eq. 17. Solid line: discontinuous profile $(U(z)$ given by M-O profile, Eq. 5, for $z \leq 0,99 h$ and $U(z)=U(h)=U_{\infty}$ for $\left.z>0,99 h\right)$; dotted line: continuous profile, Eq. 15 , with $c_{0}=0.025 U_{\infty}$; dashed line: continuous profile with $c_{0}=0.1 U_{\infty}$ (the value employed by Weng 1997). For each $L_{m o}, u_{*}$ is computed by the proposed method $\left(u_{*}=0.111 \mathrm{~m} / \mathrm{s}\right.$ for $L_{m o}=20 \mathrm{~m}$ and $u_{*}=0.191 \mathrm{~m} / \mathrm{s}$ for $L_{m o}=600 \mathrm{~m}$ )

The need to decrease the value of $c_{0}$ in Eq. 15 stems from the behaviour of $h_{m}$ and $\Delta S$ as a function of stability, a problem that was apparently overlooked by Weng. As shown in Fig. 2, a smaller value of $c_{0}$ leads to a more abrupt transition of the mean velocity from the Monin-Obukhov profile in the surface layer to its constant value aloft. However, larger values of $c_{0}$ lead to anomalous variations of $h_{m}$ and $\Delta S$ with stability. Furthermore, if $u_{*}=$ const., the behaviour of $h_{m}$ and $\Delta S$ are not satisfactory even with $c_{0}=0.025 U_{\infty}$ (as will be seen), which emphasizes the importance of allowing $u_{*}$ to vary.

Equation 11 shows that the behaviour of the streamwise velocity fluctuation, and hence also of $\Delta S$ (see Eq. 13) is highly dependent on the depths $h_{i}$ and $h_{m}$. Therefore, a correct estimation of these quantities is very important. However, because Eq. 15 has the same form as the M-O profile, Eq. 5, near the surface, $h_{i}$ does not depend on $u_{*}$. The situation is different for $h_{m}$, however. Figure 3 shows $h_{m}$ as a function of $1 / L_{m o}$, computed with prescribed or variable $u_{*}$ for the two values of $c_{0}$. When $u_{*}$ is prescribed $\left(=0.2 \mathrm{~ms}^{-1}\right), h_{m}$ varies discontinuously for both values of $c_{0}$ in the region of weak stability. The location of the jump depends on $c_{0}$ : for $c_{0}=0.025 U_{\infty}$ it occurs near $1 / L_{m o} \approx 0.05 \mathrm{~m}^{-1}\left(N_{\infty} \approx 0.0046 \mathrm{~s}^{-1}\right)$, while for $c_{0}=0.1 U_{\infty}$ it occurs for $1 / L_{m o} \approx 0.0024 \mathrm{~m}^{-1}\left(N_{\infty} \approx 0.0025 \mathrm{~s}^{-1}\right)$ and is approximately twice larger in magnitude. There is no reasonable physical reason for the existence of these jumps. By contrast, for the $h_{m}$ curve obtained using $u_{*}$ computed by the method proposed here with $c_{0}=0.025 U_{\infty}$, the jump is eliminated. Note that the variation of $h_{m}$ obtained with the same method but with the M-O wind profile, Eq. 5, (not plotted in Fig. 3) is practically coincident with that using the continuous profile, Eq. 15. This reinforces the idea that the method proposed here preserves the essential aspects of M-O scaling. 


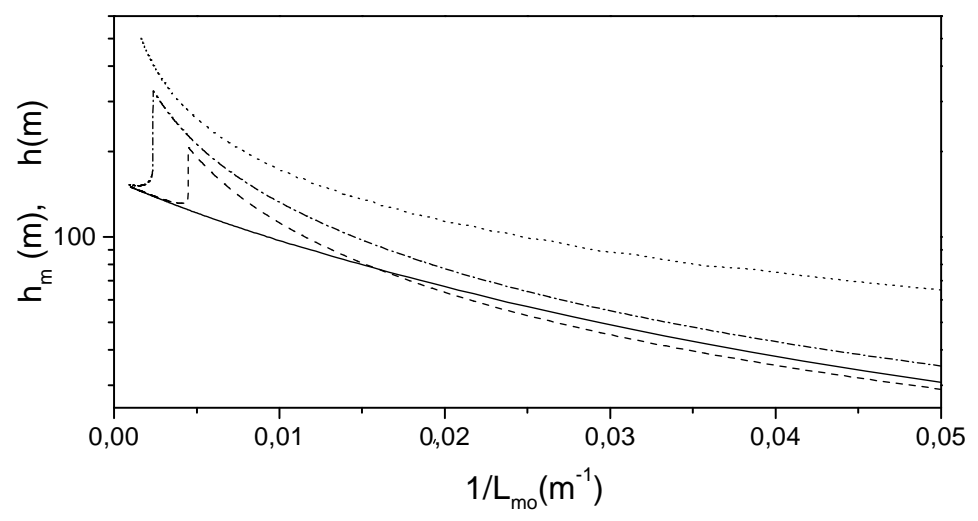

Fig. 3 Middle-layer height $\left(h_{m}\right)$ and boundary-layer height $(h)$ as a function of stability. Solid line: computed $u_{*}$ with $c_{0}=0.025 U_{\infty}$; dashed line: prescribed $u_{*}$ with $c_{0}=0.025 U_{\infty}$; dash-dotted line: prescribed $u_{*}$ with $c_{0}=0.1 U_{\infty}$; doted line: $h$ calculated using Zilitinkevich's relation, Eq. 17

Figure 3 also shows values of $h$ calculated using Zilitinkevich's relation, Eq. 17. As can be seen $h$, like the $h_{m}$ determined with the proposed method, monotonically decreases with $1 / L_{m o}$. This behaviour makes sense, because the BL becomes shallower with increasing stability. In particular, the height at which the turbulent shear stress reduces to a given fraction of its surface value should always decrease with $L_{m o}$.

According to Wyngaard (1975), $h$ may be defined as the height where the shear stress reduces to 5 percent of its surface value. In accordance with M-O similarity theory, the shear stress in a BL with unidirectional flow may be calculated as $\tau=\rho \kappa z u_{*} \Phi_{m}^{-1} \partial U / \partial z$, where $\rho$ is the density. In Fig. 4, values of $h$ are shown using the theoretical model with either prescribed or variable $u_{*}$, and Wyngaard's definition. These are compared with values of $h$ computed directly from Zilitinkevich's similarity relation, Eq. 17. It can be seen that Zilitinkevich's curve and the one obtained with the method proposed here (modified HLR theory with computed $u_{*}$ using $c_{0}=0.025 U_{\infty}$ ) are almost coincident, revealing that the corresponding velocity profile is realistic. In contrast, the curves of $h$ obtained with the same theory but prescribed $u_{*}$ (either for $c_{0}=0.025 U_{\infty}$, or $c_{0}=0.1 U_{\infty}$ as in Weng 1997) depart significantly from Zilitinkevich's curve: the values of $h$ obtained with $c_{0}=0.025 U_{\infty}$ considerably underpredict Zilitinkevich's expression and those calculated with $c_{0}=0.1 U_{\infty}$, while in slightly better agreement, intercept Zilitinkevich's curve, suggesting some physical inconsistency. 


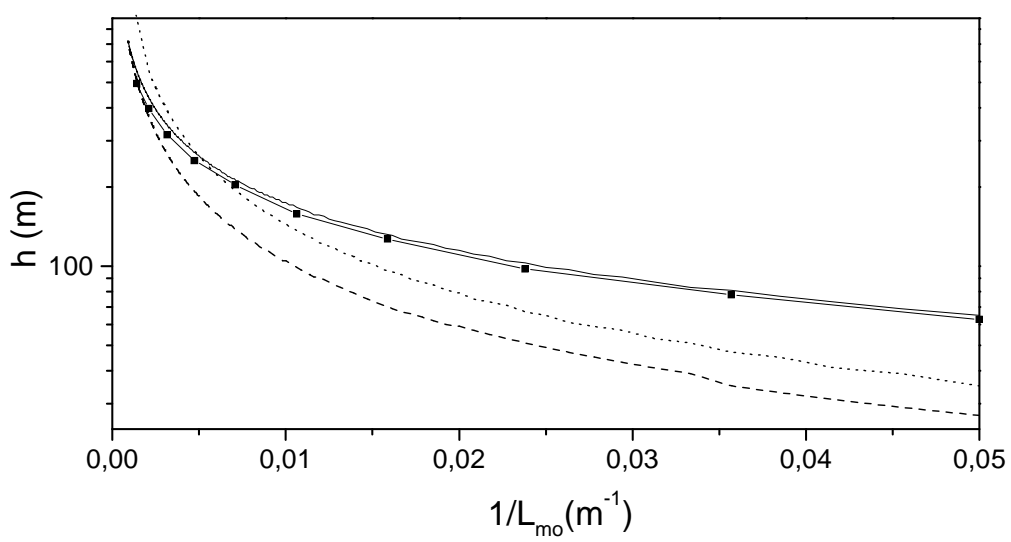

Fig. 4 Boundary layer depth $(h)$ as a function of stability. Symbols: $h$ from Zilitinkevich, Eq. 17; solid line: $h$ calculated according to Wyngaard (1975), using a computed $u_{*}$ and $c_{0}=0.025 U_{\infty}$; dashed line: $h$ as in the previous case but with prescribed $u_{*}$; dotted line: $h$ as in the previous case but with $c_{0}=0.1 U_{\infty}$

The observations reported Coppin et al. (1994) may be used to assess the theoretical results produced by the model with the friction velocity computed using the new method. Figure 5 shows the fractional speed-up ratio $(\Delta S)$ as a function of $1 / L_{m o}$, for different heights, from the different versions of the theoretical model, from the numerical model FLEX and from the observations of Coppin et al. (1994). Despite the considerable dispersion of the observations, the results produced by the proposed method compare better, in all cases, both with the numerical model and with the field data. More importantly, the speed-up values computed with a prescribed $u_{*}$ exhibit unphysical jumps in the weak to moderate stability range, which are bigger for $c_{0}=0.1 U_{\infty}$ than for $c_{0}=0.025 U_{\infty}$. These jumps of $\Delta S$ correspond directly to the jumps in $h_{m}$ observed in Fig. 3, due to the strong dependence of $\Delta S$ on $h_{m}$. The improvement in the fit between the theoretical model and the data is clearer in moderate to high stability values $\left(1 / L_{m o}>0.015 \mathrm{~m}^{-1}\right)$, where the results with prescribed $u_{*}$ clearly underpredict the observed speed-up. Except for the case $z=16 \mathrm{~m}$, where the numerical results slightly under-predict the observations, the FLEX model always appears to perform better than the theoretical model. Despite the existing differences, the improvement in the performance of the theoretical model due to the new method of calculating $u_{*}$ is striking. In general, this new method produces results much closer to both the field data and the numerical simulation results. 


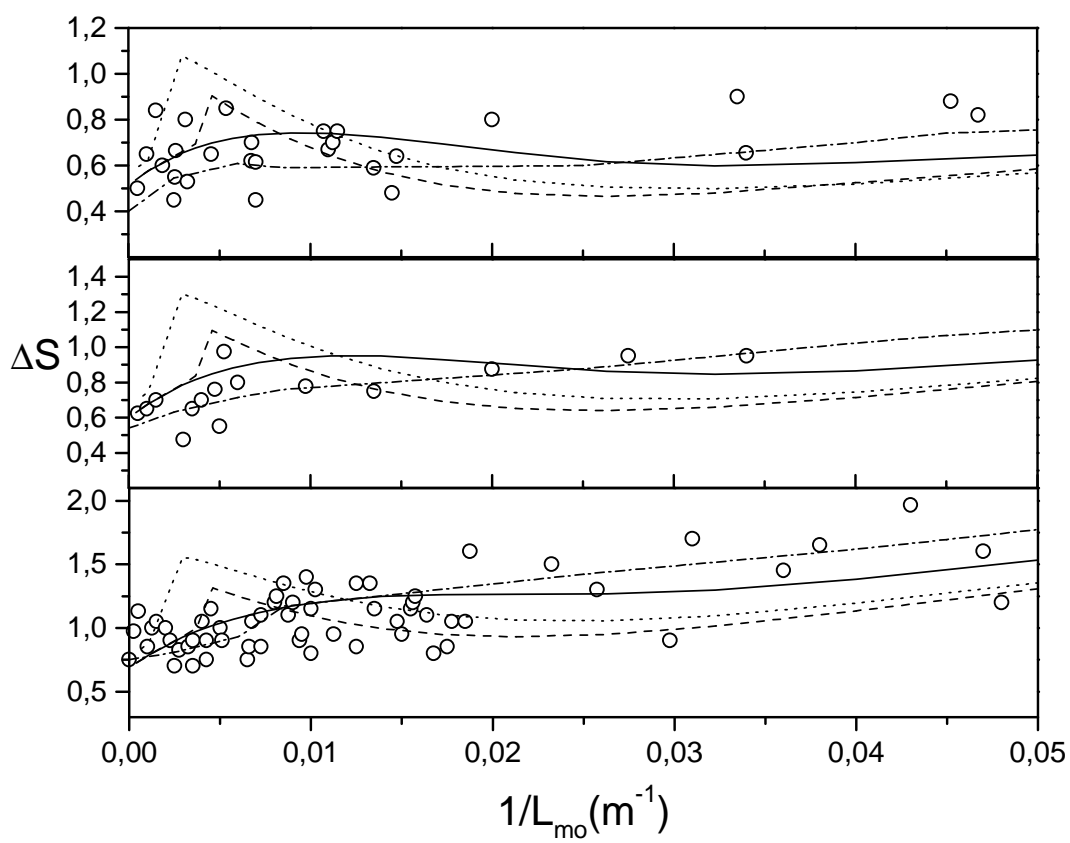

Fig. 5 Variation of the fractional speed-up ratio $(\Delta S)$ as a function of stability above the hill crest, at the heights $z=(2,8,16) \mathrm{m}$ (from bottom to top). Solid line: computed $u_{*}$ with $c_{0}=0.025 U_{\infty}$; dashed line: prescribed $u_{*}$ with $c_{0}=0.025 U_{\infty}$; dotted line: prescribed $u_{*}$ with $c_{0}=0.1 U_{\infty}$; dash-dotted line: FLEX model; symbols: observations from Coppin et al. (1994)

Additional support for the approach proposed here is given by Fig. 6, which shows a comparison of vertical profiles of the streamwise velocity perturbation for $L_{m o}=24 \mathrm{~m}$, using the two alternative methods of obtaining $u_{*}$. Also shown are results from the numerical model FLEX and observation data from Coppin et al. (1994). Both theoretical profiles were computed for $c_{0}=0.025 U_{\infty}$. Note that $d$, the zero plane displacement, takes a value of $0.35 \mathrm{~m}$, estimated from the field data. Despite the considerable differences between the profiles obtained with the theoretical model and the observations near the surface, which also occur in Weng (1997) and seem to be an intrinsic feature of his modified solution, it is clear that the proposed method, with variable $u_{*}$, leads to better results. It could be enlightening to make an analogous comparison for $L_{m o} \approx 200 \mathrm{~m}$, because the jump in $\Delta S$ occurs at this value of $L_{m o}$ for the prescribed $u_{*}$ case but, unfortunately, there are no experimental data available for these conditions. As could be observed in Fig. 5, the analytical $\Delta S$ curves obtained with $c_{0}=0.025 U_{\infty}$ and $c_{0}=0.1 U_{\infty}$ are very close together for $L_{m o}=24 \mathrm{~m}\left(1 / L_{m o} \approx 0.04 \mathrm{~m}^{-1}\right)$ at all considered heights. For that reason, the $u$ ' profile obtained with the theoretical model and $c_{0}=0.1 U_{\infty}$, is not presented in Fig. 6. 


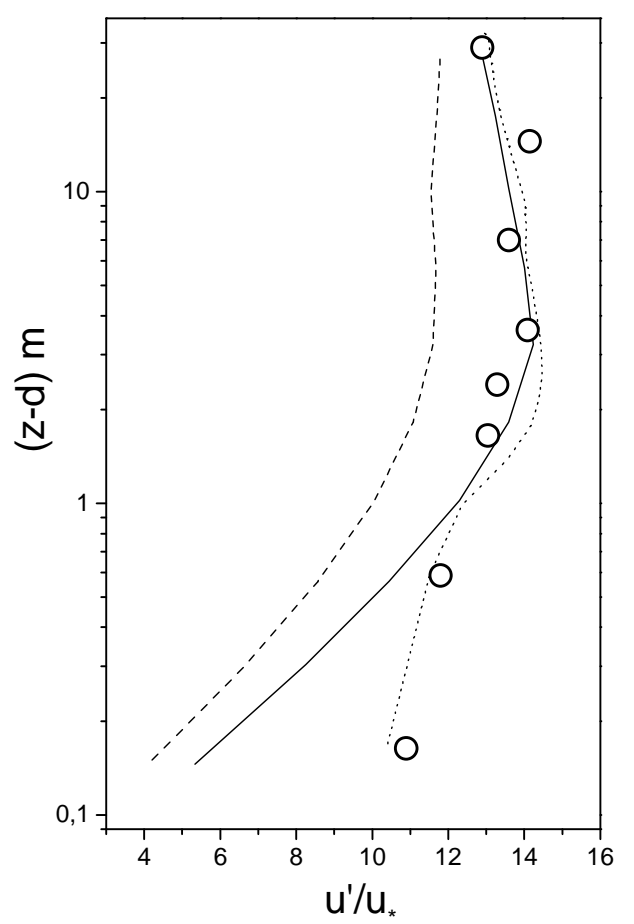

Fig. 6 Profiles of the streamwise velocity perturbation normalized by $u_{*}$, for $L_{m o}=24 \mathrm{~m}$, using $c_{0}=0.025 U_{\infty}$. Solid line: computed $u_{*}$; dashed line: prescribed $u_{*} ;$ open circles: observations from Coppin et al. (1994); dotted line: numerical results from FLEX

\section{Conclusions}

The theory developed by HLR for boundary-layer flow over hills and extended by HRB to take into account the effects of stratification has been modified by Weng (1989), with a reformulation of the inner layer solution. Additionally, Weng (1997) incorporated in this theory a vertical wind profile that makes a smooth transition from the strongly sheared surface layer flow to a constant wind speed aloft. It was found here that, while such a profile is desirable from a theoretical and practical point of view, it leads to inconsistent behaviour of some of the flow properties. This behaviour manifests itself through a jump in the flow speed-up as a function of $\mathrm{M}-\mathrm{O}$ length scale, which in turn results from the dependence of the height of the middle layer $h_{m}$ on stability.

The reason for the anomalous behaviour of the solution was related with an inconsistent prescription of the friction velocity, which was taken as constant and, therefore, was independent of static stability. In this study it was shown that it is possible to obtain a consistent definition of the friction velocity, incorporating theoretical results from Zilintikevich (1972). It was also found that the smoothness of the transition from the $\mathrm{M}-\mathrm{O}$ velocity profile, in the surface layer, to constant flow in the outer layer, must be tuned in order to avoid the same anomalous behaviour.

The proposed method leads to an overall improvement of the solution, guaranteeing a consistent variation of the boundary layer structure, and of the flow speed-up, with stability. It also leads to significantly better agreement of the theoretical model with a set observations of boundary layer flows at different stability regimes, reported by Coppin et al. (1994), and with results of a numerical model also presented in this paper. 


\section{Acknowledgements}

This work has been supported by Fundação para a Ciência e Tecnologia (FCT) under project AWARE, PTDC/CTE-ATM/65125/2006.

\section{References}

Akylas E, Tsakos Y, Tombrou M, Lalas DP (2003) Considerations on minimum friction velocity. Quart J Roy Meteorol Soc 129:1929-1944.

Argain JL (2003) Modelação de Escoamentos Atmosféricos: Efeitos Orográficos e de Camada Limite (in Portuguese). Ph.D. Thesis, Universidade do Algarve.

Barker EH, Baxter TL (1975) A Note on the Computation of Atmospheric Surface Layer Fluxes for use in Numerical Modelling. J Appl Meteorol 14:620-622.

Belcher SE, Newley TMJ, Hunt JCR (1993) The drag on an undulating surface induced by the flow of a turbulent boundary layer. J Fluid Mech 249:557-596.

Belcher SE, Wood N (1996) Form and wave drag due to stably stratified turbulent flow over low ridges. Quart J Roy Meteorol Soc 122:863-902.

Businger JA, Wyngaard JC, Izumi Y, Bradley EF (1971) Flux Profile Relationships in the Atmospheric Surface Layer. J Atmos Sci 28:788-794.

Coppin PA, Bradley EF, Finnigan JJ (1994) Measurements of flow over an elongated ridge and its thermal stability dependence: The mean field. Boundary-Layer Meteorol 69:173-199.

Dyer A.J (1974) A review of flux-profile relationships. Boundary-Layer Meteorol 7:363-372.

Högström U (1996) Review of some basic characteristics of the atmospheric surface layer. Boundary-Layer Meterol 78:215-246.

Hunt JCR, Leibovich SL, Richards KJ (1988a) Turbulent Shear Flows Over Low Hills, Quart J Roy Meteorol Soc 29:16-26.

Hunt JCR, Richards KJ (1984) Stratified Airflow over One or Two Hills. Boundary-Layer Meteorol 30:223-2.

Hunt JCR, Richards KJ, Brighton PWM (1988b) Stably Stratified Flow Over Low Hills. Quart J Roy Meteorol Soc 114:859-886.

Jackson PS, Hunt JCR (1975) Turbulent Wind Flow Over a Low Hill. Quart J Roy Meteorol Soc 101:929-955.

Klug W (1967) Determination of Turbulent Fluxes of Heat and Momentum from the Wind Profile. Quart J Roy Meteorol Soc 93:101-104.

Lopes AS, Palma JMLM, Castro FA (2007) Simulation of the Askervein Flow. Part 2: Large Eddy Simulations. Boundary-Layer Meteorol 125:85-108.

Louis JF (1979) A parametric model of vertical eddy fluxes in the atmosphere. Boundary-Layer Meteorol 17:187-202.

Patancar SV (1980) Numerical heat transfer and fluid flow. Hemisphere Publishing Corporation.

Paulson CA (1970) The mathematical representation of wind speed and temperature profiles in the unstable atmospheric surface layer. J Appl Meteorol 9:857-861.

Rossby CG, Montgomery RB (1935) The layers of frictional influence in wind and ocean currents. Pap Phys Oceanogr Meteorol 3(3):101 pp.

Taylor P, Teunissen H (1985) Askervein Hill Project: Report on the September/October 1983 Main Field Experiment. Internal Rep. MSRB-84-6, Atmos. Environ. Service, Downsview, Ontario, Canada.

Teixeira MAC, Miranda MA, Argain JL, Valente MA (2005) Resonant gravity wave drag enhancement in linear stratified flow over mountains. Quart J Roy Meteorol Soc 131:17951814.

Teixeira MAC, Miranda PMA, Argain JL (2008) Orographic gravity waves in two-layer sheared flows: critical level effects and drag enhancement. Quart J Roy Meteorol Soc In press.

Ting CL, Hay DR (1975) On the Heat Flux and Friction Velocity in Free Convection Near the Ground. J Atmos Sci 32:637-638.

Tjernstrüm M, Smedman A (1993) The Vertical Turbulence Structure of the Coastal Marine Atmospheric Boundary Layer. J Geophys Res 98:4809-4826.

Weber RO (1999) Remarks on the Definition and Estimation of Friction Velocity, Boundary-Layer Meteorol 93:197-209.

Weng W (1989) Turbulent air flow and fluxes over low hills. Ph.D. Thesis, Cambridge University.

Weng W (1997) Stably stratified boundary-layer flow over low hills: A comparison of model results and field data. Boundary-Layer Meteorol 85, 223-241. 
Weng W, Chan L, Taylor PA, Xu D (1997) Modelling stably stratified boundary-layer flow over low hills. Quart J Roy Meteorol Soc 123:1841-1866.

Wyngaard JC (1975) Modelling the planetary boundary-layer extension to the stable case. Boundary-Layer Meteorol 9:441-460.

Yagüe Y, Viana S, Maqueda G, Redondo JM (2006) Influence of stability on the flux-profile relationships for wind speed, $\phi_{m}$, and temperature, $\phi_{h}$, for the stable atmospheric boundary layer. Nonlinear Processes in Geophysics 13:185-213.

Zilitinkevich S (1972) On the determination of the height of the Ekman boundary layer. BoundaryLayer Meteorol 3:144-145. 


\section{Figure Captions}

Fig. 1 Friction velocity $\left(u_{*}\right)$ as a function of $1 / L_{m o}$. Solid line: computed $u_{*}$; dashed line: prescribed $u_{*}\left(u_{*}=0.2 \mathrm{~m} / \mathrm{s}\right)$

Fig. 2 Upstream velocity profiles, normalized by $U_{\infty}=U(h)$, for (a) $L_{m o}=20 \mathrm{~m}$, (b) $L_{m o}=600 \mathrm{~m}$. The height is normalized by $h$, estimated from Eq. 17. Solid line: discontinuous profile $(U(z)$ given by M-O profile, Eq. 5, for $z \leq 0,99 h$ and $U(z)=U(h)=U_{\infty}$ for $\left.z>0,99 h\right)$; dotted line: continuous profile, Eq. 15 , with $c_{0}=0.025 U_{\infty}$; dashed line: continuous profile with $c_{0}=0.1 U_{\infty}$ (the value employed by Weng, 1997). For each $L_{m o}, u_{*}$ is computed by the proposed method $\left(u_{*}=0.111 \mathrm{~m} / \mathrm{s}\right.$ for $L_{m o}=20 \mathrm{~m}$ and $u_{*}=0.191 \mathrm{~m} / \mathrm{s}$ for $L_{m o}=600 \mathrm{~m}$ )

Fig. 3 Middle-layer height $\left(h_{m}\right)$ and boundary-layer height $(h)$ as a function of stability. Solid line: computed $u_{*}$ with $c_{0}=0.025 U_{\infty}$; dashed line: prescribed $u_{*}$ with $c_{0}=0.025 U_{\infty}$; dash-dotted line: prescribed $u_{*}$ with $c_{0}=0.1 U_{\infty}$; doted line: $h$ calculated using Zilitinkevich's relation, Eq. 17

Fig. 4 Boundary layer depth $(h)$ as a function of stability. Symbols: $h$ from Zilitinkevich, Eq. 17; solid line: $h$ calculated according with Wyngaard (1975), using a computed $u_{*}$ and $c_{0}=0.025 U_{\infty}$; dashed line: $h$ as in the previous case but with prescribed $u_{*}$; dotted line: $h$ as in the previous case but with $c_{0}=0.1 U_{\infty}$

Fig. 5 Variation of the fractional speed-up ratio $(\Delta S)$ as a function of stability above the hill crest, at the heights $z=(2,8,16) \mathrm{m}$ (from top to bottom). Solid line: computed $u_{*}$ with $c_{0}=0.025 U_{\infty}$; dashed line: prescribed $u_{*}$ with $c_{0}=0.025 U_{\infty}$; dotted line: prescribed $u_{*}$ with $c_{0}=0.1 U_{\infty}$; dash-dotted line: FLEX model; symbols: observations from Coppin et al. (1994)

Fig. 6 Profiles of the streamwise velocity perturbation normalized by $u_{*}$, for $L_{m o}=24 \mathrm{~m}$, using $c_{0}=0.025 U_{\infty}$. Solid line: computed $u_{*}$; dashed line: prescribed $u_{*} ;$ open circles: observations from Coppin et al. (1994); dotted line: numerical results from FLEX 
Figures

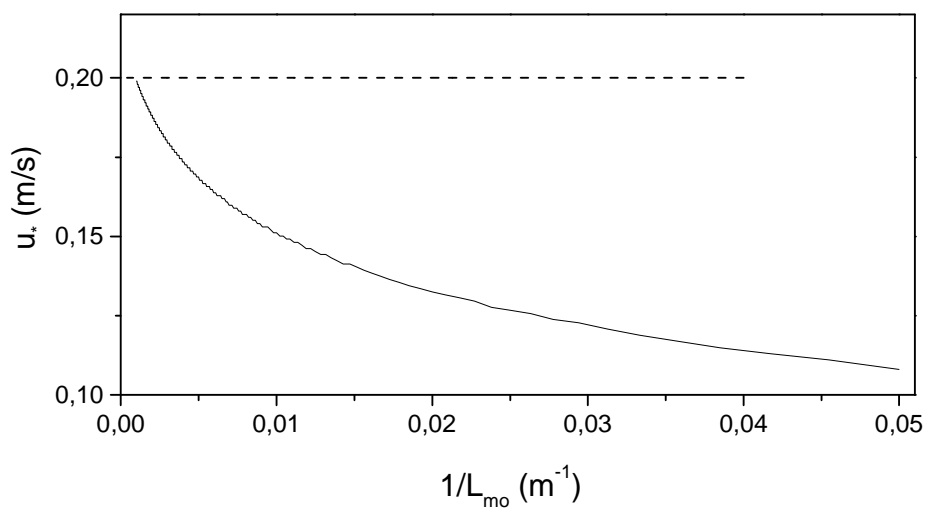

Fig. 1

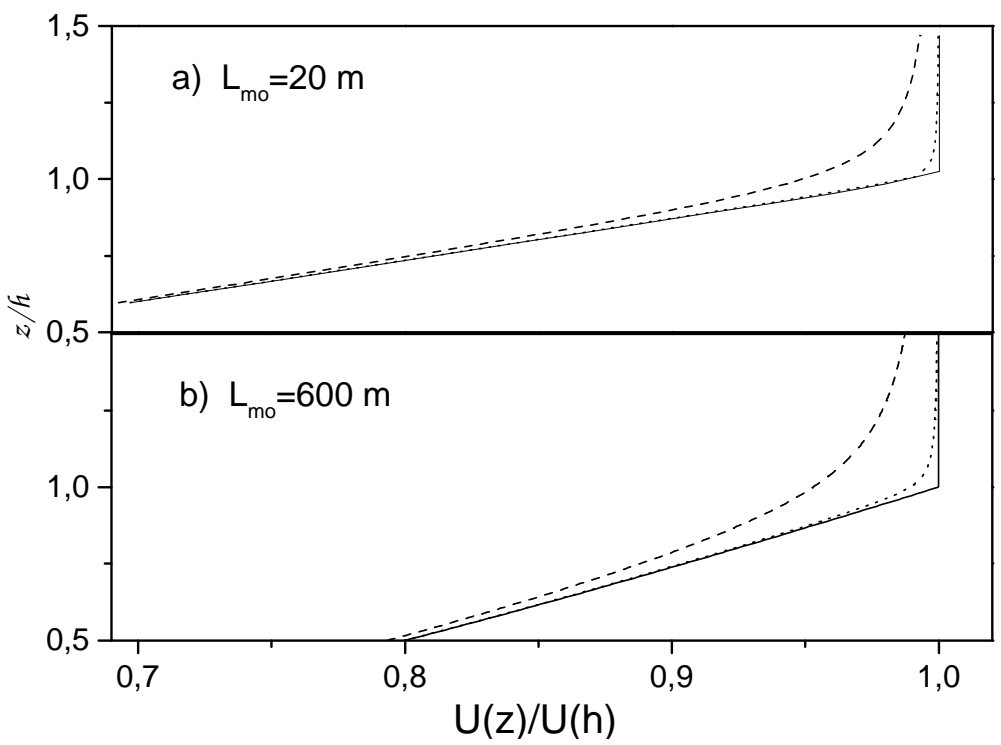

Fig. 2 


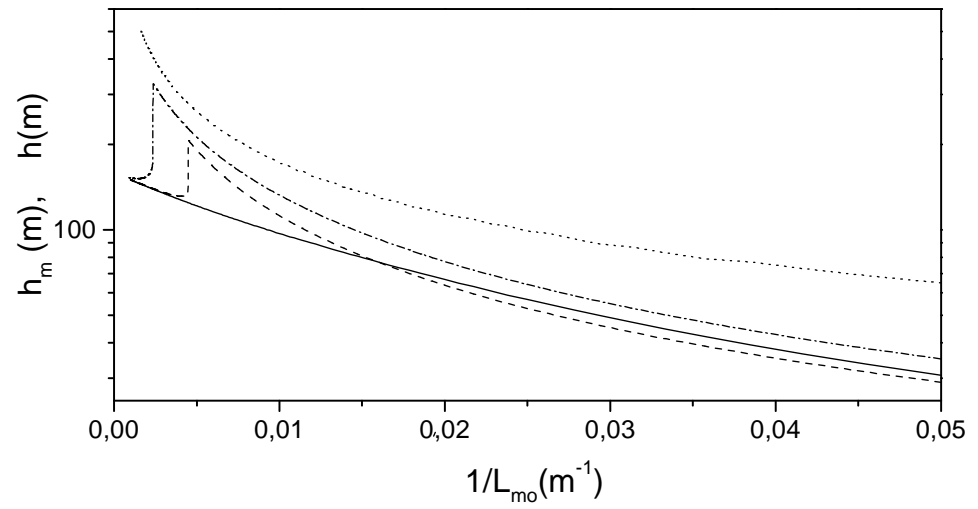

Fig. 3

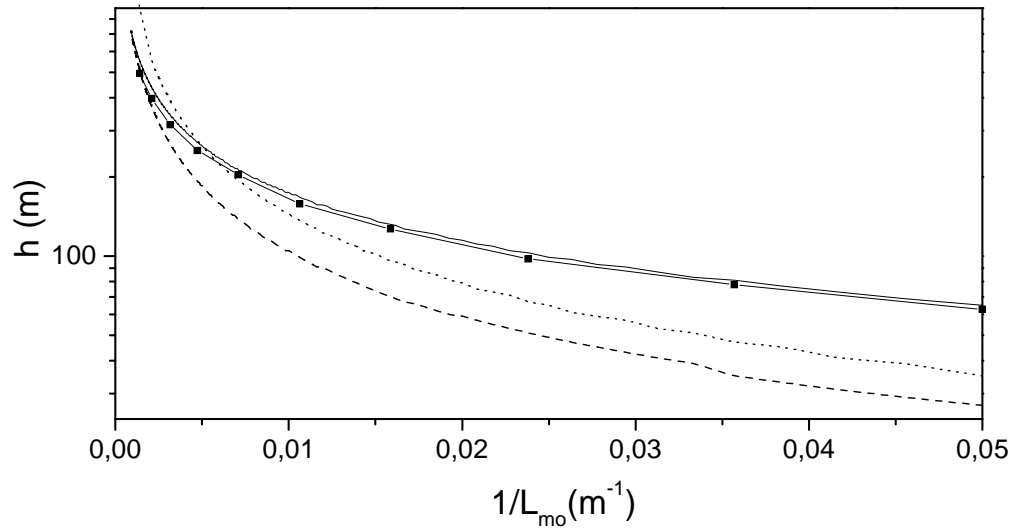

Fig. 4 


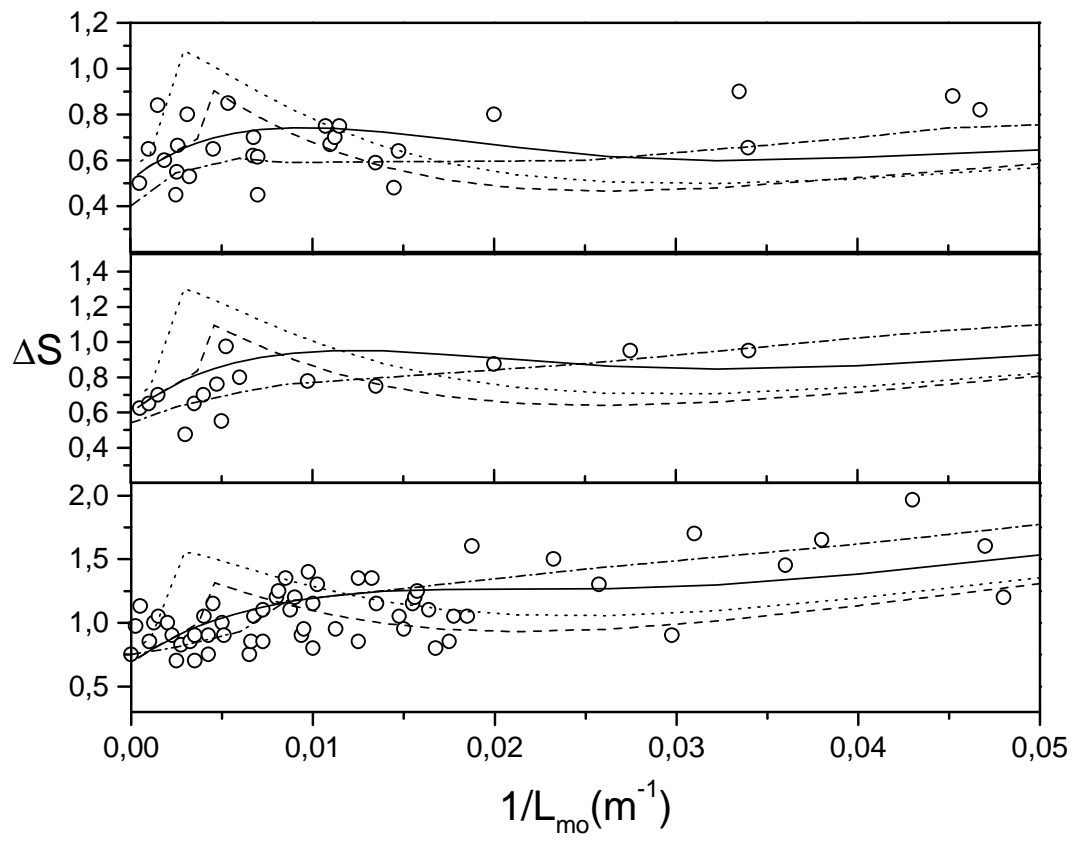

Fig. 5

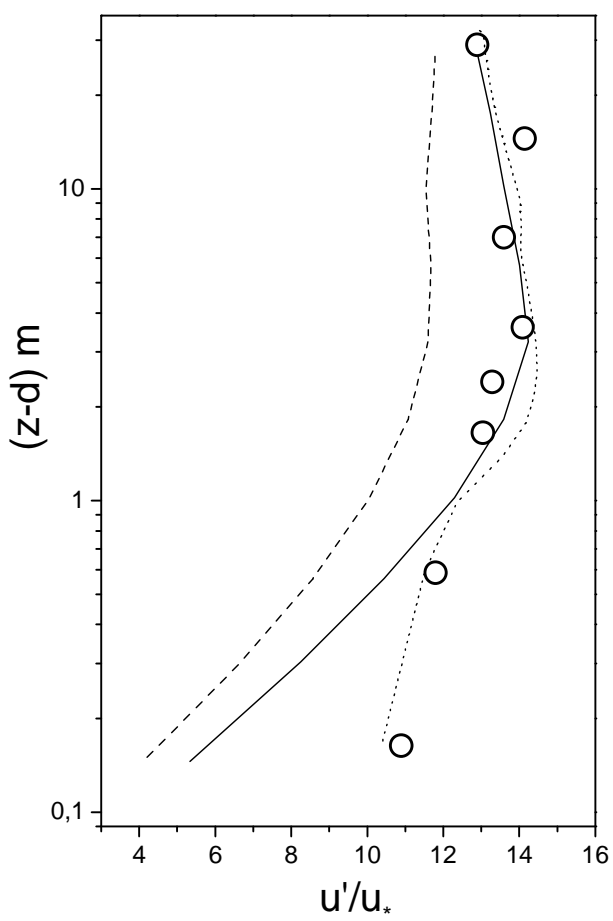

Fig. 6 